\title{
ENTRETIEN AVEC PIERRE HASSNER
}

\author{
réalisé par Henriette AssÉo et Élisabeth GESSAT-ANSTETT \\ CERI, 26 octobre 2001
}

HA : Dans quel contexte avez-vous écrit ce texte ?

PH : Le point de départ a été un colloque sur le cosmopolitisme, plutôt centré sur la culture, organisé à l'initiative de la revue Nexus. Il s'intitulait : «Y a-t-il encore un monde pour les cosmopolites? »J'ai proposé un papier sur le rapport entre le cosmopolitisme en tant qu'éthique avec Kant pour référence et en tant que culture esthétique, tout en y introduisant la politique, qui m'est plus familière. Par ailleurs, j'ai fait une conférence à la New School for Social Research de New York, où j'ai proposé ce thème que j'ai complété. Et enfin, j'ai participé, il y a quatre ou cinq ans, à un projet sur la démocratie cosmopolitique : je réfléchissais sur la question des réfugiés comme problème posé au cosmopolitisme en faisant référence à Hannah Arendt. La personne déplacée est pour elle le personnage le plus représentatif de notre temps (la question de la statelessness se trouve là). Je faisais aussi référence à la philosophie politique d'Emmanuel Kant, en reprenant la formule selon laquelle le citoyen d'un État doit avoir conscience d'appartenir à une communauté d'une autre dimension. C'était pour moi l'occasion de souligner que l'Europe pouvait avoir pour vocation de donner une communauté à ceux qui n'en ont pas, donc aux réfugiés qui l'ont perdue, ou encore aux Tsiganes qui demandent à être reconnus comme citoyens européens.

HA : Revenons à ces deux notions différentes de « cosmopolitisme » et d' « être cosmopolite ». Il y a là une part importante de l'héritage philosophique kantien, qui implique de se référer à un cosmos qui ne soit pas un chaos, c'està-dire à un ordre du monde qui ne recoupe pas les ordres mondiaux politiques des relations internationales mais qui se fonde sur un enjeu de civilisation, sur un ordre qui, au XVIII ${ }^{\mathrm{e}}$ siècle, était bel et bien européen. Or cette dimension européenne est désormais très diffuse, votre propos se situe à une échelle mondiale. Cette échelle renvoie à l'idée que vous vous faites d'une globalisation qui n'est pas exclusivement économique. Mais un second thème intervient à 
l'intersection du cosmopolitisme kantien et de ces phénomènes de worldlessness et de statelessness pointés par Arendt. Car appartenir à un ordre cosmopolite à l'heure actuelle recoupe-t-il le fait d'être citoyen du monde? En relisant récemment Primo Levi, j'ai été frappée de ce qu'il dit des camps. Les camps ne renvoient pas pour lui à un endroit cosmopolite mais à un tohu-bohu assourdissant, à la fois marqué par la promiscuité et le désordre absolu, l'impossibilité de s'entendre, qu'il oppose à la tour de Babel. On revient à cette opposition du cosmos et du chaos.

PH : Je dirais qu'il y a deux oppositions implicites qui se rejoignent sans se recouper totalement, entre le cosmopolitisme intellectuel ou culturel et le cosmopolitisme politique. Une notion familière aux Grecs veut que toute cité soit en quelque sorte dans sa propre caverne, qu'elle ait ses propres mythes et que l'Homme soit membre de sa cité. Mais le philosophe, lui, est citoyen de l'univers, il a accès à l'universel même s'il y a une tension entre l'ordre politique et l'ordre universel. Socrate accepte de boire la ciguë, car le philosophe doit accepter de jouer le jeu de la cité. Mais lui et ses disciples représentent une sorte de contre-cité, ils sont les citoyens de l'universel tout en ayant les pieds dans leur propre cité. À la conférence dont j'ai parlé précédemment, le poète d'origine polonaise, Adam Zagajewski, affirmait d'une façon aristocratique les vertus de l'exil qui vous fait participer de plusieurs univers. Or je maintiens l'opposition entre le cosmopolite de vocation aristocratique, qui peut voyager et correspondre, et le réfugié qui n'a aucune envie d'être cosmopolite, qui voudrait rester sur sa terre et qui en est chassé soit par la faim, soit par la guerre civile, soit par la persécution. Il se retrouve cosmopolite malgré lui. Pour illustrer le propos de ma conférence, j'avais pris l'exemple de l'aide ménagère d'une de mes amies à Berlin, qui était bosniaque, de Srebrenica. Ses parents ne voulaient absolument pas bouger, ils ne voulaient même pas être réinstallés à Tuzla, parce que leur patrie, c'était Srebrenica et ils espéraient toujours y retourner. Et puis il y avait ses enfants, qui étaient arrivés en Allemagne et avaient appris l'allemand, mais ne pouvaient pas y rester. Le seul endroit où ils pouvaient aller, c'était l'Australie. Elle partait donc pour l'Australie avec ses fils, bien contente d'avoir un endroit où l'on voulait bien l'accueillir. Ce qui me frappe toujours, c'est l'image du réfugié en orbite, lorsqu'on est de plus en plus éconduit et de moins en moins accueilli. Ce que je voulais souligner c'est cette opposition entre un cosmopolitisme positif des citoyens du monde, et un cosmopolitisme forcé, négatif, celui de l'exilé, du réfugié, de l'expulsé qui essaye partout de jeter des racines, ou qui, au contraire, reste en diaspora.

La grande question est la suivante : le cosmopolitisme est-il fait pour des élites aristocratiques, tandis que la plupart des gens restent dans un ordre politique lié par définition à un enracinement, à une différence entre nous et les autres ? Et puis, les cosmopolites s'inspirent-ils de l'idée positive d'un ordre politique ou 
intellectuel ? Ou bien le cosmopolitisme moderne n'est-il qu'un cosmopolitisme par défaut, produit par les éclatements nationaux ?

Il reste une dernière question et à laquelle je suis incapable, en l'état, de répondre. La situation de déracinement, de flottement peut-elle amener quelque chose de positif, peut-on en tirer une éthique ? Existe-t-il des rapports entre les gens déracinés, qui ne s'expriment pas sur le mode traditionnel de l'opposition entre les membres de la communauté et les autres - les barbares et les ennemis et qui ne renvoient pas non plus à l'appartenance à un royaume alternatif, comme le dit de façon très belle Machiavel, lorsque, après avoir passé la journée avec les paysans crottés, il rentre dans son cabinet, se pare de ses plus beaux atours pour être enfin dans son vrai pays, où il discute avec les grands penseurs de l'Antiquité et rejoint la république des lettres ? Est-ce que, par delà le dénuement ou le flottement du cosmopolitisme, on peut tirer quand même une éthique de la solidarité, à travers cette idée de « solitaire-solidaire » que l'on retrouve chez Camus, dans L'Homme révolté par exemple ? Le cosmopolitisme aujourd'hui est-il simplement négatif? Chez Arendt, «statelessness» peut être illustré de façon banale par l'exemple des détenteurs du passeport Nansen, de ceux qui n'ont pas d'État, à qui l'on donne des papiers pour qu'ils puissent circuler. Pour elle, il y avait avant notre époque quelque chose de l'ordre du cosmos des Grecs, puis une création historique s'y est ajoutée, quelque chose d'ordre matériel qui donnait une stabilité au monde par rapport auquel les vicissitudes individuelles pouvaient prendre sens, alors que maintenant l'homme en est réduit à quelque chose de biologique et de consumériste. Il n'existe plus d'intention de créer une œuvre ni de faire partie d'un cosmos ordonné.

Dans la différence qu'elle pose entre statelessness et worldlessness, stateless renvoie à la questions des papiers. En français, on le traduit par apatride, ce qui prend un sens un peu différent car l'expression anglaise est plus exacte. C'est le propos du chapitre de The Origins of totalitarianism où elle évoque la fin des États-nations et de la thèse de Marie-Claire Caloz-Tschopp sur les Sans-États dans la pensée d'Arendt. Certaines formulations d'Hannah Arendt, critiquées par Alain Renaut dans La Philosophie du droit, semble poser que finalement, sans l'existence d'une communauté, les Droits de l'homme n'existent pas. C'est aussi ce qu'évoque Michael Ignatieff dans son petit ouvrage The Need of strangers (La Liberté d'être humain) : quel devoir avons-nous par delà les frontières, par delà les communautés, qu'est-ce que l'homme sans sa communauté ? Il y a dans ce livre un très beau chapitre sur le Roi Lear, qui, une fois qu'il est dépouillé de ses atours, de son trône, est là nu, et l'on se demande ce qui reste de sa personne ou de son humanité ? Qu'est-ce que l'homme? Arendt en est là. Pour elle, les événements de ce siècle nous ont montré que les Droits de l'homme ne sont pas grand-chose s'il n'y a pas une communauté dont on fait partie et qui vous respecte ; sans cela, on n'est plus rien. On n'a 
plus le droit d'avoir des droits. L'important, c'est d'avoir une communauté où l'on a le droit de parler.

\section{EGA : D'où vient la notion de rooted-cosmopolitism?}

PH : La notion vient du philosophe Mitchell Cohen, qui essaie de concilier cosmopolitisme et communauté en disant qu'il faut un cosmopolitisme enraciné, un ancrage dans sa culture propre, mais en même temps reconnaître l'autre comme autre. C'est pourquoi je n'aime pas la pensée de Carl Schmitt, l'autre n'est pas mon ennemi, mais nous ne sommes pas non plus tous pareils. Pour Cohen, il faut accepter l'idée que l'autre a quelque chose de commun avec nous mais qu'il est aussi différent. Je dis que, de plus en plus, on peut avoir l'inverse : on perd des racines, mais on ne devient pas universaliste pour autant. Je reprends volontiers la formule qu'Ignatieff a extraite de Freud sur le « narcissisme des petites différences ». Plus on devient semblable et plus on insiste sur ses différences. Au fond, les Croates et les Serbes ne sont pas si différents, mais ils se construisent par opposition les uns aux autres. Le danger vient de la tentation de reconstruire son identité dans l'hostilité à l'autre par peur de la perdre. Pour Tocqueville et d'autres, la démocratie est à la fois le temps de l'individualisme et celui de l'absence d'individualité. Les gens deviennent de plus en plus semblables et de plus en plus isolés. Il pensait que Pascal ne pourrait pas être un penseur de l'Âge démocratique. Dans les années 1950, lorsque j'étais étudiant, il y avait un best-seller sociologique américain intitulé The Lonely Crowd. Son auteur David Riesman développait la même idée. Il parlait de la pseudo-personnalisation de la publicité. Par exemple, on vous suggère d'acheter un parfum parce qu'il exprimera votre personnalité. Mais en réalité, il s'agit du même parfum pour tout le monde.

EGA : Le cosmopolitisme enraciné doit-il être nécessairement territorial ? Les deux communautés emblématiques que vous prenez en exemple, les Tsiganes et la figure du Juif errant, ouvrent un champ immense des possibles.

$\mathrm{PH}$ : En effet, je ne crois pas que la déterritorialisation conduise forcément à la désappartenance, mais c'est un sujet de débat. Une question identique a été posée par le sionisme. L'identité juive requiert-elle la possession d'une terre historique ? Theodor Herzl, le fondateur du sionisme, n'était pas religieux. Il ne s'attachait pas à l'idée de la terre de la Bible, mais plutôt au mouvement des nationalités du XIX ${ }^{\mathrm{e}}$ siècle ; pour être une nation, il faut avoir son territoire. Cette idée contredit à la fois l'opinion selon laquelle c'est le territoire de la Bible et pas un autre qui revient aux Juifs, et celle qui veut que l'essence du peuple juif est d'être enraciné dans le Livre ou la Loi et non dans un territoire, que l'on peut être aussi bien Juif en Amérique latine ou en Afrique. 
EGA : Mais Arendt conçoit la communauté comme un objet politique, un ordre national. Or les dernières années du $\mathrm{XX}^{\mathrm{e}}$ siècle nous ont montré que les communautés qui pouvaient agir dans le sens de la défense ou de la revendication de droits avaient tendance à devenir a-nationales. C'est peut-être là que les formulations d'Arendt gardent une certaine richesse.

PH : Une formule toute simple serait d'envisager que l'Homme a des droits en tant qu'Homme et qu'il ne peut en jouir qu'à l'intérieur d'un État ou d'une communauté. Mais c'est l'hypothèse de la fin de l'État-nation qui intéresse Arendt ; elle est interpellée par ces grands mouvements du début du Xx siècle, panslavisme, pangermanisme, impérialisme en Afrique, qui débordent les cadres fixés et les jeux d'action/interaction qu'elle affectionne. Elle y voit les prémices du totalitarisme. Alors où va-t-on après ? Quel est l'au-delà de ce cadre national ? C'est là que se trouve l'ambiguiité. Car elle s'enthousiasme pour tous les mouvements tels que la Commune, les conseils ouvriers hongrois de 1956 ou les événements de Mai 1968, lorsque se recrée un espace public. Elle recherche finalement l'existence d'un espace public, qu'elle ne définit pas très clairement. Je pense que les initiatives comme le passeport Nansen sont un début qui donne à ceux qui n'ont rien un minimum de droits. Par ailleurs, il me semble qu'une communauté des «sans-communautés » peut avoir un sens proche de celui qu'évoque Jan Patočka quand il parle de « la communauté des ébranlés ». Mais Arendt garde une position un peu ambiguë par rapport à ça. Elle rapproche de façon un peu audacieuse la notion de statelessness, qui conserve une dimension très concrète, du concept un peu heideggérien de worldlessness ( «absence de monde »). Il n’y a plus de consensus, il n’y a plus de religion commune, nous sommes livrés à des processus qui nous dépassent, incompréhensibles, c'est la fin d'un monde stable et cohérent. Pour elle, le sort concret du Juif expulsé est une image, et ce qu'il exprime le mieux, c'est la condition métaphysique de l'Homme moderne. C'est l'image du Juif errant. La revue qui avait organisé le colloque avait mis ainsi en épigraphe une citation du livre de Franz Rosenzweig, L'Étoile de la rédemption, une belle phrase sur les Juifs qui ont le Livre au lieu d'avoir la terre. Il dit : « Nous avons nos racines en nous-mêmes et c'est comme cela que nous traversons le monde en transportant nos racines. » En un sens, c'est aussi quelque chose de très concret. La géographie et l'histoire nous ont appris qu'il y avait les peuples sédentaires et les peuples nomades, mais Salman Rushdie dit que l'homme n'est pas un arbre, il a des pieds pour s'en aller. À ce sujet, on peut opposer deux acceptions de la nostalgie : celle de la poésie populaire roumaine, exploitant le thème du dor - la nostalgie du foyer - à travers la figure du berger qui, dans la montagne, pense à son village, à ses parents et à sa bien-aimée; et celle du norvégien qui a un mot pour exprimer la nostalgie du lointain et des horizons infinis. Il y a cette dualité dans les sociétés et dans l'Homme entre un instinct d'enracinement et une tentation ou une réalité de l'errance. 
EGA : Ces communautés, s'il fallait les regarder d'un double point de vue politique et culturel, sont-elles en mesure d'offrir un avenir à ces individus et à ces groupes, jetés sur les routes ou, comme vous le dites, perpétuellement en orbite, qui se posent dans un aéroport pour en décoller sans jamais avoir été accueillies?

PH : C'est toute la question de la nature de ces communautés. Est-ce que le regroupement des personnes, détenteurs d'un passeport Nansen, est une communauté ? Est-ce que l'Europe pourrait servir à quelque chose en offrant un espace communautaire à ceux qui n'en ont pas ? C'est la question. Est-ce qu'on recrée des communautés volontaires ? On va vers un tout autre problème, où réside l'une des sources du totalitarisme ou du communisme. C'est le problème qu'Olivier Roy approche de manière intéressante quand il analyse l'origine de l'islamisme, même si je pense que son point de vue est excessif, selon lequel il n'y a pas d'internationale islamique et c'est un peu partout la nation qui l'emporte sur l'Islam. Mais il s'intéresse depuis plusieurs années aux phénomènes qui émergent selon lui en réaction à la globalisation, où des générations déracinées, installées plutôt en Occident, s'inventent une communauté, un Islam cosmopolite où ils trouvent un asile circonstanciel.

HA : Il semble même que les terroristes qui ont agi aux États-Unis représentent une sorte de mythe cosmopolite dévoyé, qui est le revers du modèle occidental. Cela m'évoque des logiques qui renvoient plutôt au monde du Komintern de 1919 et à l'imminence de la révolution mondiale.

$\mathrm{PH}$ : Avec toutefois ce thème obsessionnel de la présence des infidèles sur la Terre sacrée, qu'ils souillent de leur présence. Ce qui les rend si dangereux c'est cette relation au suicide dans la mesure où l'au-delà leur est garanti par l'Islam. Mais il est certain que cette dimension de déracinement au milieu de l'Occident donne une autre communauté qui n'est pas territoriale, ni nationale.

HA : Mais il me semble qu'il n'est pas contradictoire d'avoir, d'une part, un repli sur le communautarisme - et donc une interprétation intellectuelle du communautarisme qu'est le multiculturalisme - et, d'autre part, un renouveau cosmopolite. Ce sont des processus parallèles qui traduisent, en termes contemporains, l'impossibilité d'exprimer une politique hégémonique de la part de nations qui ne sont plus dominantes et qui, entrées en phase de repli, sont incapables d'avoir une politique impériale affirmée comme telle. Le problème fondamental c'est qu'à l'origine l'ordre cosmopolite dont rêvent Kant et les Lumières est une inscription dans le monde bien délimité de la civilisation, dans le monde policé de la civilité, et une poussée vers l'universel. Une possibilité d'articulation entre les deux existait à la fois à titre individuel et collectif. Or, à l'heure actuelle, émerge une sorte d'éclatement entre des conceptions 
individuelles, qui relèvent de l'éthique, et des conceptions collectives, qui sont d'ordre politique, une séparation entre l'éthique et le politique. Les tentatives de retour en force d'un droit cosmopolite, à travers l'idée d'un arbitrage international comme celui du Tribunal international, ne me paraissent pas être portées par une vision cohérente du cosmopolitisme. Il me semble que cette vision n'est pas cohérente parce qu'il y a un chaînon manquant, qui existait chez les Grecs, comme vous l'avez évoqué, et au XVIII' siècle : c'est l'idée d'une civilisation commune. À l'heure actuelle, même l'Occident, lorsqu'il se pense en terme de nations riches, ne se conçoit pas en tant que civilisation commune face au reste du monde. On évoque l'idée d'un « choc des civilisations » alors que personne n'est capable de s'identifier à une civilisation commune, qui ne soit pas réduite à une culture de la consommation mondialisée.

PH : C'est un problème qui m'est familier, même si je ne suis pas en mesure d'y apporter de réponse. J'ai tendance à répondre à cela un peu comme Glucksman ou Kouchner : je ne sais pas ce qu'est le bien, mais je sais ce qu'est le mal. Le seul fondement d'une morale commune c'est ce qu'on a connu, l'Holocauste, la Shoah. L'idée du «plus jamais ça » a entraîné la floraison des ONG, orphelines du marxisme et de la «realpolitik», pour reprendre une formule que j'utilise souvent. Après le Vietnam, il est difficile de croire à la politique traditionnelle des États nationaux, et après le communisme, il est difficile de croire aux lendemains qui chantent. En revanche, la morale de l'extrême urgence dit qu'il est mal de torturer les gens; ce point de vue est soumis à toutes sortes de critiques, qui disent que c'est du sentiment, de l'immédiat, un processus de victimisation qui empêche d'aller au fond des causes sociales des problèmes.

Ce qui me parle, ce sont des situations qui ressemblent à cela. Alors je ne propose pas de grands fondements métaphysiques de la morale, et c'est aussi pour cela que je m'attache à Kant. Il propose une conception différente de celles des Anciens, de Hegel ou de Marx. Néanmoins, chez Kant, il y a l'esquisse d'une philosophie de l'histoire, où la guerre, les questions de différence de langues et de religions ont pu être nécessaires, mais où il existe malgré tout un plan caché de la nature et, finalement, une unification de l'espèce.

Kant n'est pas pour un État mondial mais pour une situation cosmopolite, où il y a des États républicains et une organisation internationale dotée d'un droit cosmopolitique. Il introduit une notion qui est encore plus parlante en allemand : celle de «droit de citoyen du monde », Weltbürgerrecht. Son projet de paix perpétuelle s'appuie sur trois acquis : l'État doit être républicain, il doit y avoir une organisation contre la guerre et il existe un droit cosmopolitique, qui n'est pas identique au droit des gens ou au droit international, mais qui est un droit de chaque individu en tant que membre de la planète. C'est bien évidemment très délicat car l'homme conserve le droit d'être accueilli partout, mais Kant est contre la colonisation, donc l'homme ne peut justifier son 
établissement dans n'importe quelles conditions. Les marxistes diront que ces grandes idées ne servent que l'idéologie de la porte ouverte.

Je trouve intéressante cette manière de marcher vers les rapports pacifiés entre États et vers quelque chose qui dépasse les États et donnerait un statut aux réfugiés. Alors, quelque chose manque. Mais Kant part de la notion morale de respect, du fait que les hommes sont inégaux en capacités, mais qu'il y a une dignité égale en chaque individu, en tant qu'il est capable de raison ou de comportement moral. Même si l'on peut dire que c'est de l'individualisme et du moralisme, c'est son point de départ; il n'a pas de vision très cohérente ni de la communauté politique, qui est simplement l'État de droit où les droits individuels sont respectés, ni de l'univers qui reste inconnu.

Quant aux Américains, ils pratiquent un kantisme du premier degré : ils prétendent dénoncer le mal, incarner une destinée manifeste et ne comprennent pas qu'on les déteste alors qu'ils se perçoivent comme porteurs d'une mission civilisatrice. Je citerai une phrase de Koestler qui disait que nous défendons une semi-vérité contre un mensonge total, en disant que l'on n'est pas la civilisation, mais qu'il y a une pluralité de cultures, de religions, de traditions. Mais, au-delà des différentes cultures, il y a des choses qui relèvent du « mal ». C'est peut-être dû à notre culture judéo-chrétienne ou à notre expérience du nazisme et du communisme. Partout il y a des gens à qui cela dit quelque chose (aux gens de la place Tien'Anmen par exemple).

Je ne peux malheureusement pas aller au-delà, mais je crois qu'on ne peut adopter l'assurance et l'optimisme naïf des Lumières; il n'est pas possible cependant d'en rester au constat du relativisme des cultures et de leurs conflits.

Kant adopte un point de vue universel qui est plus intransigeant que celui de Hegel. Il déteste voir les gens s'agenouiller, attitude contraire à la dignité de l'Homme. Même s'il reste des choses confuses dans sa Géographie, où il reprend les préjugés de son temps, il prend la mesure de l'influence de Rousseau sur lui. Il dit que ce dernier lui a appris le «prix de l'Homme », et qu'il considère que tous ses travaux théoriques n'ont aucune valeur, s'ils ne servent pas à donner à l'Homme la conscience de sa dignité et de ses droits à la protéger. Alors que les pensées de Hegel sont très centrées sur l'Europe, avec cette idée que les barbares devraient être civilisés un jour. Ce sont aussi les conceptions du $\mathrm{XIX}^{\mathrm{e}}$ siècle, où l'autodétermination reste réservée aux gens qui en sont capables.

EGA : Si pour Kant le cosmopolitisme est pour tout le monde, les poncifs de son temps qu'il restitue dans sa Géographie sont-ils le support de jugements de valeur? Par ailleurs, ne pensez-vous pas, qu'au-delà de cet universel cosmopolite il puisse y avoir des enjeux de civilisation, qui ramèneraient le cosmopolitisme à un simple idéal en conférant une place prépondérante aux ordres nationaux ou impériaux du XIX ${ }^{\mathrm{e}}$ siècle? 
PH : Dans Les Géographies de l'Esprit, Marc Crépon se bat avec cette idée. Pour lui, Kant distingue les peuples selon leurs degrés divers d'ouverture à l'universalité. Mais Kant pose comme un fait que tel peuple est barbare, tel autre est superficiel, qu'à tel autre on ne peut pas faire confiance. Alors que pour Hegel l'opposition est plus tranchée entre l'Europe et les autres.

Kant a cette phrase dans le Projet de paix perpétuelle où il dit que c'est grâce au développement du commerce qu'aujourd'hui une violation des Droits de l'homme à un bout du monde est ressentie partout. D'après son Idée d'une histoire universelle d'un point de vue cosmopolitique, il existe entre les États une telle interdépendance économique que, lorsqu'il se passe quelque chose dans l'un, les autres s'estiment fondés à proposer leur arbitrage et à s'en mêler. Il refuse l'opposition nette entre intérieur et extérieur et l'idée d'une souveraineté absolue. Même s'il ne va pas plus loin. Alors que chez Hegel, la tradition impériale est beaucoup plus proche. Chez Kant, on a l'impression que les empires, les conquêtes, les guerres, les dominations sont nécessaires au cours de l'histoire, mais on arrive finalement à une sorte d'égalité en droit. Son idée c'est que, grâce au commerce, le mouvement de l'histoire va dans ce sens-là. Pour reprendre la distinction que fait Marx entre socialisme utopique et socialisme scientifique, il pense qu'il y a des forces à l'œuvre dans l'histoire, qui vont dans ce sens-là. Cela soulève un autre point. Puisqu'il envisage l'idée d'un plan caché de la nature, on se demande ce qu'est cette nature : la Providence, Dieu, l'Histoire ? Il pose une idée qui sera développée par Hegel et Marx, celle de la ruse de la raison. Il dit une chose que reprend Arendt, et qui n'est pas chez Marx et Hegel ; selon lui, il est incompréhensible que les hommes souffrent pour un but qui ne sera réalisé que des siècles et des siècles plus tard. Pourquoi cette nature de l'Homme, d'un Homme qui ne réalise sa nature qu'à l'échelle de l'espèce dans un horizon lointain et qui est incapable de le faire au niveau de la vie individuelle?

Est-ce que le cosmopolitisme est simplement un idéal culturel, philosophique ou est-ce qu'il peut servir un idéal politique ? L'ouvrage de Maria Daraki, Une religiosité sans Dieu, étudie le cosmopolitisme des stoïciens et la controverse sur sa nature de proposition politique ou d'image. Ces philosophes de l'Antiquité posent que le monde est comme une grande cité dont les hommes sont membres et qu'il existe une hiérarchie naturelle. Il y a pourtant débat sur la question de savoir s'ils proposaient un projet de cité universelle dépassant les États ou bien si leur réflexion s'arrêtait à la métaphore de la grande cité commune, l'ordre de chaque cité devant être un reflet particulier de cet ordre global cosmique. 